\title{
Dynamical masses of two young globular clusters in the blue compact galaxy ESO 338-IG04 ${ }^{\star}$
}

\author{
G. Östlin ${ }^{1}$, R. J. Cumming ${ }^{1}$, and N. Bergvall ${ }^{2}$ \\ 1 Stockholm Observatory, AlbaNova University Center, Stockholms Center for Physics, Astronomy and Biotechnology, \\ Roslagstullsbacken 21, 10691 Stockholm, Sweden \\ e-mail: [ostlin@;robert]@astro.su.se \\ 2 Uppsala Astronomical Observatory, Box 515, 75120 Uppsala, Sweden
}

Received 2 November 2005 / Accepted 26 September 2006

\section{ABSTRACT}

\begin{abstract}
We present high-resolution échelle spectroscopy, obtained with the UVES spectrograph on ESO/VLT, of two luminous star clusters in the metal-poor blue compact galaxy ESO 338-IG04 at a distance of $37.5 \mathrm{Mpc}$. Cross-correlating with template stars, we obtain line-of-sight velocity dispersions of 33 and $17 \mathrm{~km} \mathrm{~s}^{-1}$. By combining with size estimates from Hubble Space Telescope images we infer dynamical masses of $1.3 \times 10^{7} M_{\odot}$ and $4.0 \times 10^{6} M_{\odot}$ for the two clusters, making them among the most massive known. The less massive cluster is the faintest cluster for which a dynamical mass has yet been obtained. In both clusters we detect Balmer absorption lines which we use to estimate their ages. From the younger ( $\sim 6 \mathrm{Myr}$ ) and more massive cluster, we detect He II $\lambda 4686$ emission of intermediate width, indicating the presence of very massive O-stars. Moreover, analysis of the [O III] $\lambda 5007$ and $\mathrm{H} \alpha$ emission lines from the region near the younger cluster indicates that it is associated with a bubble expanding at $\sim 40 \mathrm{~km} \mathrm{~s}^{-1}$. We also see from the Na ID absorption lines indications of neutral gas flows towards the younger cluster. We compare the dynamical masses with those derived from photometry and discuss implications for the stellar initial mass function (IMF) in each cluster. Both clusters are compatible with rather normal IMFs which will favour their long-term survival and evolution into massive bona fide globular clusters.
\end{abstract}

Key words. galaxies: evolution - galaxies: individual: ESO338-IG04 (Tol 1924-416) - galaxies: starburst - galaxies: star clusters galaxies: stellar content

\section{Introduction}

Super star clusters (SSCs) are important sites for star formation in starburst galaxies (Arp \& Sandage 1995; Meurer et al. 1995), and may be the progenitors of globular clusters (GCs). Different observational techniques have been used to investigate the nature and history of these objects. High resolution imaging, notably with the Hubble Space Telescope (HST), has been used to collect photometry and to estimate the sizes of many SSCs (Whitmore 2003). Photometry, in combination with spectral evolutionary synthesis models, has also been used to estimate cluster ages and masses (Östlin et al. 1998). While many such photometric masses have been determined, only a small fraction of extragalactic SSCs and GCs have dynamical mass estimates. Taken together, measurements of the photometric and dynamical masses can allow constraints on the stellar initial mass function (IMF) and the dynamical state in these objects.

The spectrum of a cluster can provide other information about the stellar population. For example, González Delgado et al. (1997) inferred a population of Wolf-Rayet stars in the massive cluster NGC 1569-A, and Origlia et al. (2001) used ultraviolet spectra to show that the same cluster has a population of O-stars.

^ Based on observations collected at European Southern Observatory, Paranal, Chile, under observing programme 65.N-0704; and on observations with the NASA/ESA Hubble Space Telescope, obtained at the Space Telescope Science Institute, which is operated by the Association of Universities for Research in Astronomy, Inc., under NASA contract NAS5-26555.
High-resolution spectra can be used to measure the velocity dispersion, and, assuming virialisation, to estimate dynamical masses for SSCs and GCs. Ho \& Filippenko (1996a,b) were first to use the cross-correlation method described by Simkin (1974) and Tonry \& Davis (1979) to measure the velocity dispersions of NGC 1569-A (giving a kinematic mass of $3.3 \times 10^{5} M_{\odot}$ ) and the SSC in NGC $1705\left(8.2 \times 10^{4} M_{\odot}\right)$. The same method has been applied to M82-F by Smith \& Gallagher $\left(2001 ; 1.2 \times 10^{6} M_{\odot}\right)$, to five clusters in NGC 4214 and NGC 6946 by Larsen et al. (2001, 2004; $0.2-1.8 \times 10^{6} M_{\odot}$ ), by Gilbert \& Graham (2002) to clusters A1, A2 and B in NGC $1569\left(3.9 \times 10^{5} M_{\odot}, 4.4 \times 10^{5} M_{\odot}\right.$ and $2.3 \times 10^{5} M_{\odot}$, respectively) and by McCrady et al. (2003) to two SSCs in M82 $\left(1.5 \times 10^{6} M_{\odot}\right.$ and $\left.3.5 \times 10^{5} M_{\odot}\right)$. Mengel et al. (2002) measured the dynamical masses of five SSCs in NGC $4038 / 4039\left(0.65-4.7 \times 10^{6} M_{\odot}\right)$ by fitting model spectra in the near-IR and optical. Maraston et al. (2004) presented a dynamical mass estimate of as much as $8 \times 10^{7} M_{\odot}$ for the cluster W3 in NGC 7252, which may possibly be a dwarf galaxy. Bastian et al. (2006) found masses of $1.6 \times 10^{7} M_{\odot}$ for both W30 in NGC 7252 and G114 in NGC 1316, and present a compilation of previously published results (their Table 6).

A possibly related class of objects are the nuclear star clusters found in many bulgeless spiral galaxies (e.g. Walcher et al. 2005), some of which appear to be very massive but which probably have more extended star formation histories than GCs and SSCs.

ESO 338-IG04, also known as Tol 1924-416, is a luminous $\left(M_{B}=-19\right)$ blue compact galaxy (BCG) - the closest in a class of galaxies that are rare in the local universe but that become 
increasingly important towards higher redshifts and the peak in the cosmic star formation rate at $z \sim 1$ (e.g. Werk et al. 2004). ESO 338-IG04 shows both vigorous star formation and a rich population of more than 100 young SSCs and old globular clusters (Östlin et al. 1998) ${ }^{1}$. Analysis of the age distribution of these clusters shows evidence that at least one strong starburst occurred in the past, a couple of Gyr ago, and the present starburst has probably been active for about $40 \mathrm{Myr}$ (Östlin et al. 2003). In addition ESO 338-IG04 hosts a population of cosmologically old (age $\leq 10 \mathrm{Gyr}$ ) globular clusters. What makes ESO 338IG04 particularly interesting as a star cluster formation laboratory is its combination of small reddening, many clusters, and a low metallicity $(12+\log (\mathrm{O} / \mathrm{H})=8.0)$. Compared to other metal-poor star-forming galaxies in the local universe (such as NGC 1569, NGC 1705, and He 2-10) the clusters in ESO 338 IG04 are more luminous and numerous. The present starburst has been triggered by a small merger or interaction with its companion galaxy (Östlin et al. 2001; Cannon et al. 2004). Hence ESO 338-IG04 offers the possibility of directly studying globular cluster formation associated with hierarchical galaxy evolution, at a low metallicity.

In this paper, we report high-dispersion échelle spectroscopy of two of these clusters, labelled Inner-\#23 and Outer-\#34 by Östlin et al. (1998). Hereafter, we refer to them simply as \#23 and \#34. Cluster \#23, a young blue cluster situated in the centre of the starburst region, is the most luminous in the whole galaxy $\left(M_{V}=-15.5\right)$. Cluster \#34 is of intermediate age, $\sim 1 \mathrm{Gyr}$, but still very luminous $\left(M_{V}=-12.8\right)$, which led Östlin et al. (1998) to suspect a mass in excess of $10^{7} M_{\odot}$. Here, we use highdispersion VLT/UVES spectroscopy to make dynamical mass estimates and new age estimates for both clusters. These two clusters were selected since they represent the most luminous of the young and intermediate age clusters, respectively, and could be observed in a single UVES slit.

The outline of the rest of the paper is as follows. In Sect. 2 we describe the observations and reductions. In Sect. 3 we show the results obtained; Sect. 3.4 in particular describes how we obtained estimates of the velocity dispersions of the clusters through cross-correlation techniques. In Sect. 4 we discuss the inferred dynamical masses and compare with results derived from photometry. Section 5 summarises our conclusions.

\section{Observations and reductions}

\subsection{UVES-spectroscopy}

Our spectra were taken on 2000 June 15 using the échelle spectrograph UVES mounted on the telescope Kueyen (UT2) of the Very Large Telescope, at the European Southern Observatory's Paranal site. The observations were performed in service mode. The seeing was typically 0 . $^{\prime} 6$.

We placed the slit at a fixed position angle of $42^{\circ} .6$ in order to capture the spectra of both cluster \#23 and \#34 in the same exposure (see Fig. 1). To minimise contamination from other sources in this crowded starburst environment, we used a slit width of 0.6 . The slit lengths used were $8^{\prime \prime}$ and $11^{\prime \prime}$ for the blue and red cameras, respectively. The atmospheric dispersion corrector in UVES was used to minimise slit losses due to differential refraction.

Using the dichroic setting DIC1 with cross-dispersers CD2 and CD3, we covered the optical spectrum from $3280 \AA$

${ }^{1}$ Following Östlin et al. (1998) we adopt a distance of $37.5 \mathrm{Mpc}$ to ESO 338-IG04.
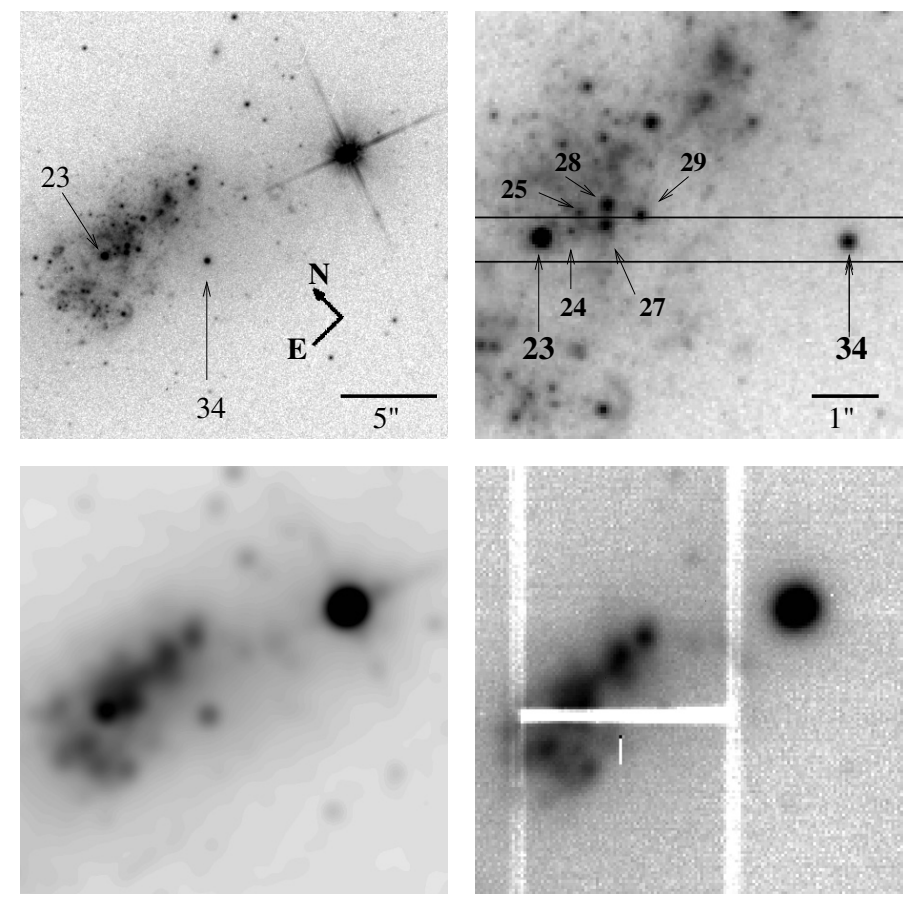

Fig. 1. Acquisition. Upper left: HST/WFPC2 image of ESO 338-IG04 in the $\mathrm{F} 814 \mathrm{~W}$ band, rotated to match the position angle used for our UVES observations. The orientation is indicated and the two main targets for the spectroscopy discussed in this paper are labelled. Upper right: enlargement of the upper left image showing the slit location, the main targets and some other nearby clusters. The width of the slit is $0.7^{\prime \prime}$. The clusters have been identified with the index number used by Östlin et al. (1998). All but \#34 belong to their "inner sample". Lower left: the image in the upper left panel, convolved with a Gaussian kernel the same size as the seeing $\left(F W H M=0{ }^{\prime} 65\right)$ to simulate the actual observing conditions. Lower right: slit view image from the red camera of UVES. The slit is the horizontal bright bar, and has a length of $11^{\prime \prime}$. The bright foreground star on the right was used for offsetting in the acquisition.

to $6660 \AA$ at a resolution of $R \sim 60000$. We binned the CCD pixels $2 \times 2$ to lower the influence of the readout noise, which resulted in a spatial scale of 0.50 for the blue arm and 0.'36 per pixel for the red arm. A total of eight 2000-s exposures were taken. The data were reduced using the UVES pipeline, with extraction and sky windows modified to allow for the extraction of two non-centred sources. Our wavelength calibration, done by comparison with ThAr lamps, should be accurate to better than $0.02 \AA$, or about $1 \mathrm{~km} \mathrm{~s}^{-1}$.

Using the same instrumental set-up, we observed five template stars (for details, see Table 1). Two of these, HR 7706 and HR 7749, were observed on 2000 April 22; the rest were observed on the same night as the cluster observations.

\subsection{Source extraction}

Special care was taken with the background subtraction, though the small size of the slit and the complex H II region emission meant that the subtraction is unreliable in the vicinity of strong emission lines. This is also due to the intrinsically different spatial distribution of nebular emission and the stellar continuum (see Östlin et al. 2003 and Fig. 9). Spectra were extracted using average extraction, centred on the continuum of the clusters. The extraction windows were 3 and 5 pixels wide for the blue and red 
Table 1. Template stars.

\begin{tabular}{llcl}
\hline \hline Designation & Type and class & Radial velocity $\left(\mathrm{km} \mathrm{s}^{-1}\right)$ & Reference $^{1}$ \\
\hline HR 6961 & K4 Ib & -2.291 & Nidever et al. (2002) \\
HR 7277 & K1 I & +2.8 & Ochsenbein (1980) \\
HR 7706 & K1 III & $+1.0 \pm 0.3$ & This work \\
HR 7749 & F5 V & -30.8 & Kharchenko et al. (2004) \\
HR 8299 & G5 III & $-57.1 \pm 0.4$ & This work \\
\hline
\end{tabular}

Note: (1) All references are to catalogues available at the VizieR website, http://vizier.u-strasbg.fr/ (Ochsenbein et al. 2000).

arm spectra, respectively. We extracted the spectra for both the clusters and the template stars in this way.

While clusters \#23 and \#34 are well-separated on the sky, light from a number of other sources was also collected by our slit (see Fig. 1). In particular, we expect a contribution from at least two more clusters from the inner sample of Östlin et al. (1998): numbers 24 and 27 (\#24 and \#27, see Fig. 1). Both are slightly offset from the centre of the slit and we estimate that about $50 \%$ of the flux from \#24 and about $15 \%$ of that from \#27 are collected by our extraction window. Both clusters are however fainter than \#23, by 4.2 and $2.1 \mathrm{mag}$, respectively, in the $F 555 W$ filter (Östlin et al. 1998). Based on this we estimate that on the order of $1 \%$ or less of the flux from \#23 has its origin in each of \#24 and \#27. Contamination from known sources should thus not be a problem.

Nevertheless, since \#23 is located in a crowded area, we also tried using narrower extraction windows (width 1 pixel for the blue arm, and 3 pixels for the red arm) with the centre offset by 1 pixel to the north-east, where the crowding is less severe (see Fig. 1).

\subsection{HST Imaging}

In addition to the UVES spectra, we used images from the Hubble Space Telescope obtained with the Planetary Camera (PC) aperture of the WFPC2 in the $F 218 W, F 336 W, F 439 W$, $F 555 W$ and $F 814 W$ passbands. These data are described fully in Östlin et al. (1998, 2003). We used these images to estimate the sizes of the two clusters, and to constrain their age and photometric mass from comparison with spectral evolutionary synthesis models. The photometry used in the current paper differs slightly from that of Östlin et al. (1998) in that we have optimised the photometric parameters specifically for these two clusters and also corrected for the charge transfer (in)efficiency of WFPC2 (Whitmore et al. 1999; Dolphin 2000) using the web-based tool CTE Tool $\# 1^{2}$. This effect is noticeable for faint sources on low backgrounds and is here important mainly for the $F 336 \mathrm{~W}$ magnitude of \#34 where it amounts to $\sim 0.06 \mathrm{mag}$.

\section{Results}

\subsection{The spectrum of cluster \#23}

Figure 2 shows parts of the spectrum of cluster \#23. The spectrum shows a stellar continuum with superimposed narrow emission lines and a few emission lines of intermediate width. The narrow emission lines come from extended emission close to the cluster. Background subtraction across this emission was not always successful, leading to the spurious absorption features seen in some of the emission lines.

\footnotetext{
2 CTE Tool \#1 by Andrew Dolphin www.stsci.edu/instruments/wfpc2/Wfpc2_cte/ wfpc2_cte_calc.html
}
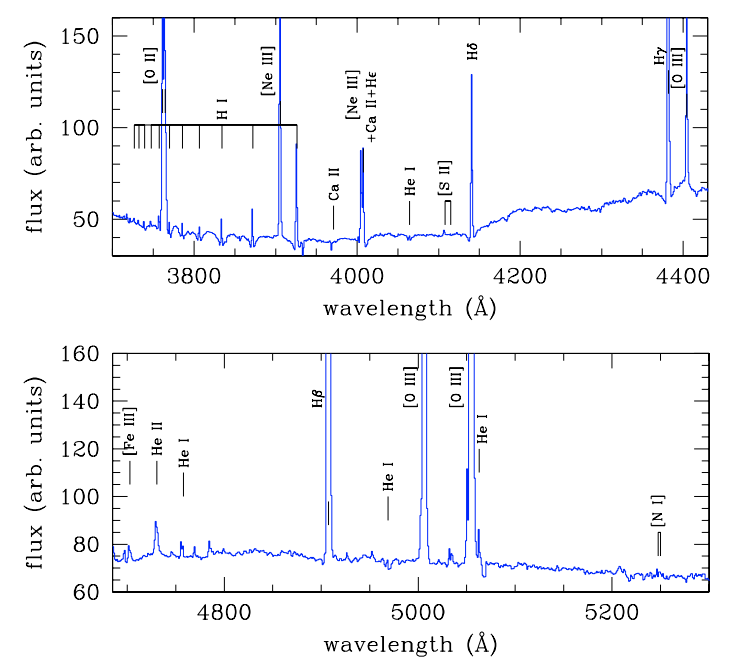

Fig. 2. Part of the spectrum of cluster \#23 with line identifications. The spectrum has not been flux-calibrated. Note the absorptions in the Balmer series and the broad emission line in He II $\lambda 4686$.

The narrow line emission presumably originates from a combination of $\mathrm{H}$ II regions and supernova remnants. We have identified lines of H I, He I, [N I], [N II], [O II], [O III], [Ne III], [S II], [S III] and [Fe III]. The ratio of [O II] $\lambda 3729$ to $\lambda 3726$ indicates a emitting electron density of around $150 \mathrm{~cm}^{-3}$.

In addition, broader emission is seen in He II $\lambda 4686$ ( $F W H M \sim 460 \mathrm{~km} \mathrm{~s}^{-1}$ ) and possibly C IV $\lambda 4658$ (blended with narrower [Fe III]). The line widths we see here are comparable to those seen in Of stars, but narrower than in Wolf-Rayet stars (cf. Nota et al. 1996).

Stellar absorption lines with broad wings are seen in the Balmer series. Narrower absorptions are seen in He I, Na I D (both at the redshift of ESO 338-IG04 and at zero redshift from the local Galactic ISM) and Ca II K. A number of other absorption features are apparent towards the red parts of the spectrum, in particular at 5168/5172 $\AA(\mathrm{Mg} \mathrm{I}), 5185 \AA(\mathrm{Mg} \mathrm{I}, \mathrm{MgH})$, and also at $6138 / 42 \AA$ and $6496 \AA$.

\subsection{The spectrum of cluster \#34}

Cluster \#34 was detected with much poorer signal-to-noise ratio than \#23. Its spectrum shows $\mathrm{Na}$ I D absorption lines, the Mg I doublet at 5168/5172 $\AA$, and a number of other features also seen in the template stars. No broad emission lines of the type seen in cluster \#23 were detected, though features with the same equivalent widths (EW) could easily be hidden in \#34's noisier spectrum. 

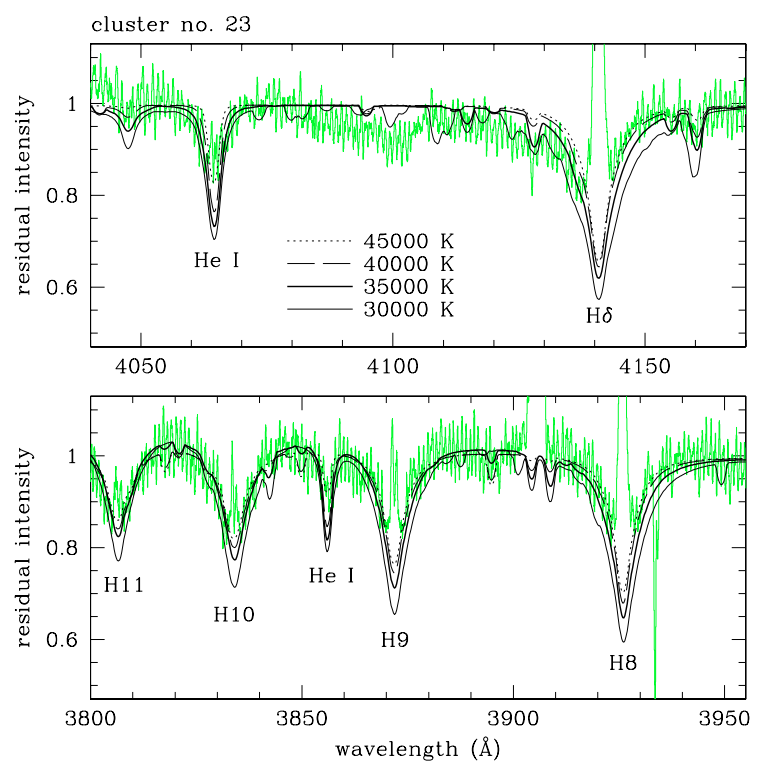

Fig. 3. Normalised spectrum of cluster \#23, with superimposed models of González Delgado \& Leitherer (1999). We show models for $\log g=$ 4.5 and effective temperatures of $30000 \mathrm{~K}$ (thin solid line), $35000 \mathrm{~K}$ (thick solid line), $40000 \mathrm{~K}$ (broken line) and $45000 \mathrm{~K}$ (dotted line). For ease of comparison, the slope of the model spectra shortward of $3940 \AA$ has been adjusted to match the data.

\subsection{Cluster ages from Balmer lines}

We have estimated the ages of the clusters by fitting the model spectra of González Delgado \& Leitherer (1999) to the Balmer and $\mathrm{He} \mathrm{I}$ line spectrum around $\mathrm{H} \delta$ and in the region from $\mathrm{H} 8$ to H11.

First we smoothed our spectra to match the resolution of the models. Comparing the models with the smoothed data, we located the combination of effective temperature and surface gravity which gave the best fit to the observed line spectrum. Since the line cores are dominated by emission in our data, we matched based on the wings of the lines.

For cluster \#23 we get $T_{\text {eff }}=3.75 \pm 0.5 \times 10^{4} \mathrm{~K}$ (corresponding to late $\mathrm{O}$ stars), $\log g=4.5-5$; and for \#34 we get $T_{\text {eff }}=1.3_{-0.3}^{+0.7} \times 10^{4} \mathrm{~K}$ (late B stars), $\log g=4.5-5$. In Figs. 3 and 4 we show some of these fits. Adopting $\log g=4.5$, we estimated the equivalent widths of the $\mathrm{H} \delta$ and $\mathrm{H} 8$ lines using the data in Tables 4 and 5 of González Delgado \& Leitherer (1999, GDL99). We obtain, for \#23, $W_{\mathrm{H} \delta}=3.6_{-0.6}^{+1.5} \AA$ and $W_{\mathrm{H} 8}=2.8_{-0.4}^{+0.7} \AA$, and for \#34, $W_{\mathrm{H} \delta}=12_{-4}^{+6} \AA$ and $W_{\mathrm{H} 8}=9_{-3}^{+3} \AA$.

Finally, we estimated the age of each cluster by comparing these equivalent widths derived from $\mathrm{H} \delta$ and $\mathrm{H} 8$ with the values for different instantaneous population ages presented by GDL99 (their Table 5, for $Z=0.001$ and a Salpeter (1955) $\mathrm{IMF}^{3}$ with mass range 1 to $80 M_{\odot}$ ). We obtain $\log t(\mathrm{yr})=6.8 \pm 0.2$ for cluster \#23, or $6_{-2}^{+4}$ Myr.

This metallicity is slightly lower than the ISM abundance of ESO 338-IG04, which could lead us to overestimate the age slightly. Moreover, one may speculate that the high dynamical mass of \#23 (see Sect. 3.7) could allow some self-enrichment with the result that this cluster would be richer in metals than its environment. On the other hand, the use of an upper mass limit of $80 M_{\odot}$, as in the GDL99 model, would lead to an underestimate of the age if the true IMF were to extend to $120 M_{\odot}$.

${ }^{3}$ We parameterise the IMF as a power law, $\mathrm{d} N / \mathrm{d} M \propto M^{-\alpha}$, where a Salpeter IMF has $\alpha=2.35$.
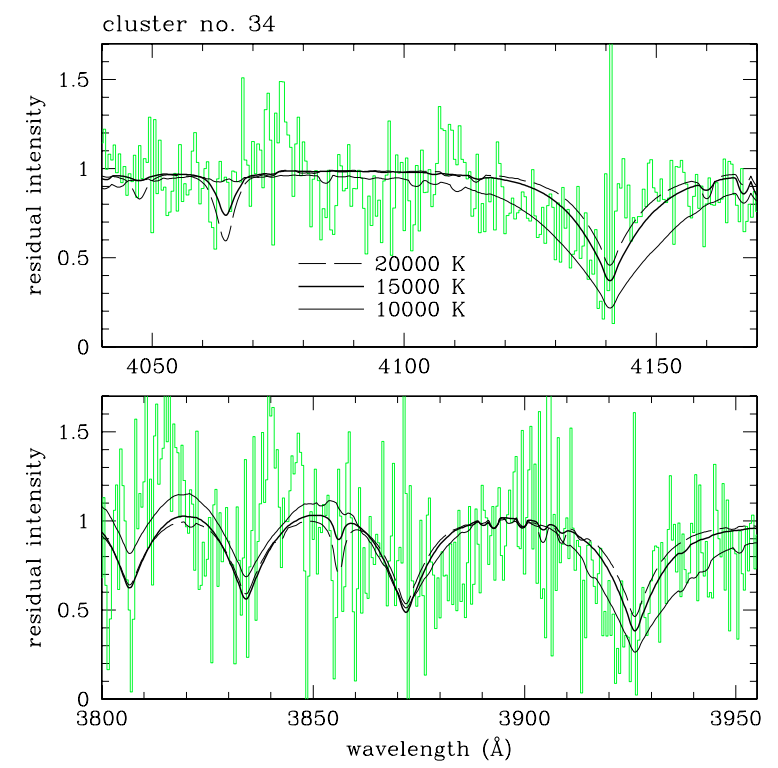

Fig. 4. As Fig. 3, but for cluster \#34. Here the models have effective temperatures of $10000 \mathrm{~K}$ (thin solid line), $15000 \mathrm{~K}$ (thick solid line) and $20000 \mathrm{~K}$ (broken line).

For the fainter cluster \#34, we cannot set very stringent limits on the age. One reason is the noisy spectrum, leading to uncertain EW estimates. In addition, the Balmer line absorption EW of a low-metallicity stellar population increases with time up to an age $\sim 1$ Gyr, after which it decreases again (Bica \& Alloin 1986). Based on the GDL99 models, the estimated EW indicates an age $\sim 0.3 \mathrm{Gyr}$, with a lower limit on the age of $50 \mathrm{Myr}$. Comparing also with the empirical $\mathrm{H} \delta$ data from Bica \& Alloin (1986), we find for \#34 an allowed age range from about 0.5 to 3 Gyr.

As a consistency check, we compared the effective temperature of the best-fitting models to the effective temperature at the main-sequence turnoff in the models of Bertelli et al. (1994). Stars at the turnoff are expected to dominate the blue light from the clusters. This estimate gives $\log t(\mathrm{yr})=6.8 \pm 0.25$ for cluster \#23. For \#34 we obtain $\log t(\mathrm{yr})=8.6 \pm 0.6$ for a low metallicity $(Z=0.001)$ and $\sim 0.5$ dex lower for a high metallicity $(Z=0.02)$.

Thus we find an age of $\sim 6 \mathrm{Myr}$ for cluster \#23, and a rather generous age span for \#34. These results are consistent with our photometric modelling (Östlin et al. 2003), which we discuss in Sect. 4.

\subsection{Cross-correlation analysis: radial velocities and line-of-sight velocity dispersions}

To estimate the radial velocities and line-of-sight velocity dispersions of the two clusters, we cross-correlated their spectra with a number of template stars, following the method of Simkin (1974), Tonry \& Davis (1979) and Ho \& Filippenko (1996a,b). For this we used the IRAF ${ }^{4}$ task fxcor.

${ }^{4}$ IRAF (Image Reduction and Analysis Facility) is distributed by the National Optical Astronomy Observatories, which are operated by the Association of Universities for Research in Astronomy, Inc., under cooperative agreement with the US National Science Foundation. 
Table 2. Starburst99 models used to test which stellar types dominate the absorption line features in the spectral region 4600-6540 A.

\begin{tabular}{llll}
\hline \hline Model & Tracks & $Z$ & $\begin{array}{l}M_{\text {up }} \\
\left(M_{\odot}\right)\end{array}$ \\
\hline P1 & Padova & 0.004 & 120 \\
P2 & Padova & 0.0004 & 120 \\
P3 & Padova & 0.004 & 20 \\
G1 & Geneva & 0.001 & 120 \\
\hline
\end{tabular}

\subsubsection{Selecting the proper template stars}

For the cross-correlation method to yield trustworthy measurements of the velocity dispersion, the absorption line spectrum should be dominated by stars with intrinsically narrow lines common to both the target and the template star. Cool giants and supergiants are likely to be the best templates.

At the age of cluster \#34, we expect the luminosity to be dominated by late-type giants, which suggests that the optimum template star should be of this type. Cluster \#23 is much younger, but following the discussion in Ho \& Filippenko (1996a), we still expect its absorption line spectrum in the visual wavelength region to be dominated by red supergiants. Examination of the high resolution spectra of local stars presented by the UVES Paranal Observatory Project (UVESPOP; Bagnulo et al. 2003) confirms that in our wavelength range, only stars of spectral type later than A are likely to contribute significantly to the observed absorption line spectrum.

As an independent quantitative check, we used Starburst 99 (Leitherer et al. 1999; see below) to calculate the contributions from stars of different spectral types to some of the well-known Lick indices (Worthey et al. 1994). Although these are defined for resolutions much lower than ours, the type of stars that dominate a particular index should also dominate the individual lines that make up the index. The cross-correlation method should also be sensitive to these lines. Equally, a spectral type that gives a negligible contribution to the indices in the visual wavelength region is unlikely to contaminate the cross-correlation signal.

At the Starburst99 website ${ }^{5}$ it is possible to run simulations that output the number of stars for each spectral type and luminosity class, as a function of age. We ran a few such models (see Table 2) and used this information, together with the luminosity and temperature relations from Drilling \& Landolt (2000) and the fitting functions of Worthey et al. (1994), to predict the relative contribution to the Lick indices from stars of different spectral types as a function of stellar population age. Model G1 has the same tracks and metallicity as the standard model used in Östlin et al. (2003) for estimating stellar populations in the clusters in ESO338-IG04. Models P1 and P2 bracket model G1 in metallicity, and also bracket the observed nebular abundance for ESO 338-IG04. Finally, model P3 has a lower upper mass limit; in Sect. 4.1 we discuss the possibility of an IMF devoid of very massive stars. All models use a lower mass limit of $0.1 M_{\odot}$ and an IMF with slope $\alpha=2.35$.

We found that for all ages, only spectral types later than $\mathrm{A}$ contribute to the indices in the spectral region 4600-6540 A. For models P1, P2 and G1 and ages younger than 4 Myr, these indices are dominated by main sequence stars of class F, G and $\mathrm{K}$. Later, red supergiants (RSGs) take over completely, and only after 15 Myr do giants and supergiants of spectral type earlier than $\mathrm{K}$ contribute. If the cluster has an IMF lacking very massive stars, as in our model $\mathrm{P} 3$, the situation is somewhat different.

\footnotetext{
5 http://www.stsci.edu/science/starburst99/
}

Table 3. Spectral regions used for cross-correlation in this paper in the rest frame of ESO 338-IG04. "G" and "R" refer to the short and long wavelength detectors, respectively, of the UVES red arm, avoiding the strongest emission lines and telluric absorption. Weaker emission lines remaining have been masked out. The subscripts " $B$ " and " $R$ " correspond to the "blue" and "red" parts of each region, respectively.

\begin{tabular}{ll}
\hline \hline Rest wavelength range $(\AA)$ & Name \\
\hline $4606-4952,5023-5552$ & $\mathrm{G}$ \\
$5023-5552$ & $\mathrm{G}_{R}$ \\
$5665-6273,6320-6544$ & $\mathrm{R}$ \\
$5665-6273$ & $\mathrm{R}_{B}$ \\
$6320-6544$ & $\mathrm{R}_{R}$ \\
\hline
\end{tabular}

Here the main sequence stars of types $\mathrm{F}$ to $\mathrm{K}$ dominate longer, up to ages of just over $10 \mathrm{Myr}$, after which RSGs take over. This is understandable since less massive stars take longer to evolve off the main sequence. Hence, if the high-mass IMF is truncated at $M_{\text {up }}=20 M_{\odot}$, our F V template star would be the best choice for cross-correlation. However, a low-resolution long-slit spectrum of \#23 taken with VLT/FORS2 (Cumming et al. 2006, in prep.) shows the presence of the Ca II near-IR triplet, with an equivalent width of several $\AA$. This indicates that red giants or supergiants must be present (Díaz et al. 1989). The Starburst99 models also predict that strong $\mathrm{Ca}$ II triplet absorption lines develop when RSGs appear.

In summary, even though \#23 is young, we can still safely assume that red supergiants dominate the absorption features we see, and that such stars should be used as template stars for cross-correlation.

Cluster \#34 is somewhere in the range 0.3 to 1.4 Gyr old, depending on metallicity (see Sects. 3.3 and 4.2). For such ages, our analysis using Starburst99 indicates that the absorption features in the visual wavelength region are dominated by red giants of luminosity classes II and III, with a contribution from main sequence stars of types F to K. Hence a mix of all the template stars in Table 1 would seem appropriate.

A remaining potential problem is that the macroturbulent broadening in our template stars may differ from the macroturbulence characteristics of the cluster stars. This difference may be of the order of $1-3 \mathrm{~km} \mathrm{~s}^{-1}$ for giants and supergiants (Gray 1981; Gray \& Toner 1987) and somewhat larger for main sequence stars (Gray 1984). Since giants and supergiants are likely to dominate the line widths in both clusters, the importance of macroturbulence will be small, and will not be considered further.

\subsubsection{Selecting the optimum wavelength region}

We used fxcor to carry out separate cross-correlations for a number of different wavelength regions.

Prior to cross-correlation, we masked out strong emission lines, telluric absorption features, and absorption in the $\mathrm{Na}$ I D lines (see discussion in Sect. 3.5 below). The spectrum of \#23 shortward of $4745 \AA$ is contaminated by nebular emission and early-type stars. For cluster \#34, this part of the spectrum has in addition poor signal-to-noise ratio. We therefore omitted this spectral region from the cross-correlation analysis.

We show the names and ranges of the spectral regions we have used in Table 3. Inspection of the spectra (see for example Fig. 5) showed that those features which are visible above the noise in the cluster spectra tend also to be present in all our template stars, though with different strengths. All 


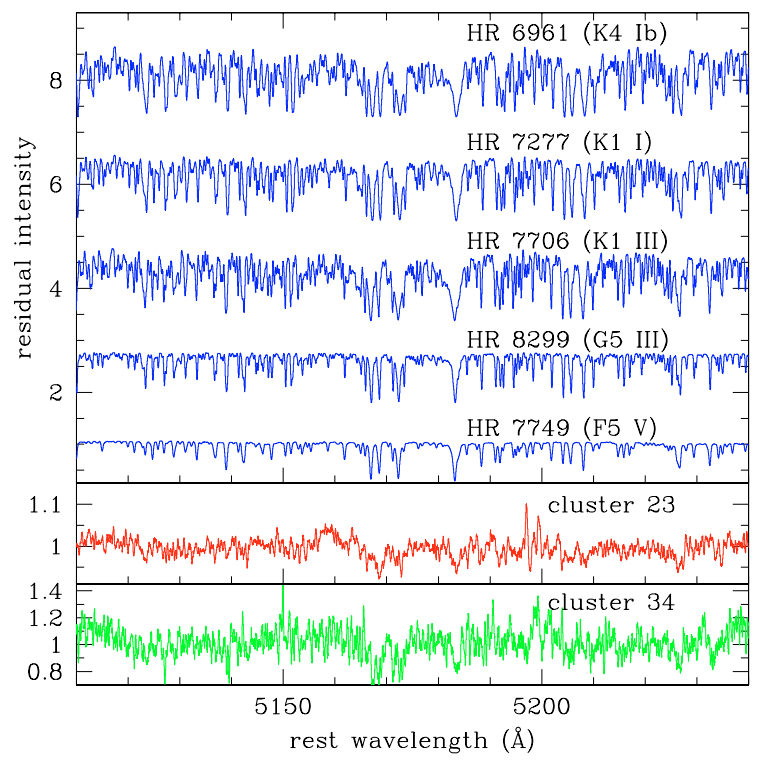

Fig. 5. Part of the spectra of the two clusters, showing the region around the $\mathrm{Mg}$ I triplet, compared to the same region in the five template stars. The spectra have been smoothed with a 9-pixel boxcar and shifted to a rest wavelength scale for clarity of presentation.

the template stars gave a positive cross-correlation signal between $4606 \AA$ and $6545 \AA$, and always at the same radial velocity, to within $\pm 4 \mathrm{~km} \mathrm{~s}^{-1}$. The blue part of region $\mathrm{G}$ (4606-4952 ̊) gave less reliable results, and will not be further considered. We found that the best cross-correlations were obtained if we filtered out the lowest frequencies in the spectrum. For this we used a ramp filter rising from zero to unity at wavenumber 90 (40) for spectral regions $\mathrm{G}$ and $\mathrm{G}_{R}\left(\mathrm{R}_{B}, \mathrm{R}\right.$ and $\mathrm{R}_{R}$ ).

For both clusters, the highest cross-correlation signal was obtained for spectral region $\mathrm{G}_{R}$, irrespective of which template star was used. Figures 6 and 7 show examples of cross-correlation functions (CCFs) for one of the template stars, HR 6961. These figures illustrate how the CCF peak amplitudes differ between wavelength regions. The CCF peak amplitudes for region $\mathrm{G}_{R}$ are always significantly larger than regions $\mathrm{G}, \mathrm{R}_{\mathrm{B}}$ and $\mathrm{R}_{R}$, which have peak values similar to that for $\mathrm{R}$.

The arguments for the choice of template star presented in Sect. 3.4.1, based on the analysis using Starburst99, are of course best founded for wavelength regions that include some of the Lick indices (Worthey et al. 1994). This is fulfilled for region $\mathrm{G}_{R}$, about half of whose wavelength range is covered by these indices. The other wavelength regions defined in Table 3 are clearly less suitable from this point of view. While region $\mathrm{R}_{B}$ contains five defined indices, two are $\mathrm{TiO}$ and one is $\mathrm{Na} \mathrm{D}$, which we have masked out to avoid contamination by interstellar absorption. The TiO lines could be problematic since they are strong only in very cool stars with later spectral type than any of our template stars, but which may nevertheless be present in our clusters (e.g. M giants and supergiants). In summary, region $\mathrm{G}_{R}$ appears to be the safest to use. It is also the wavelength region which contains most of apparent absorption features and for which the strength of the cross-correlation data is clearly the strongest.

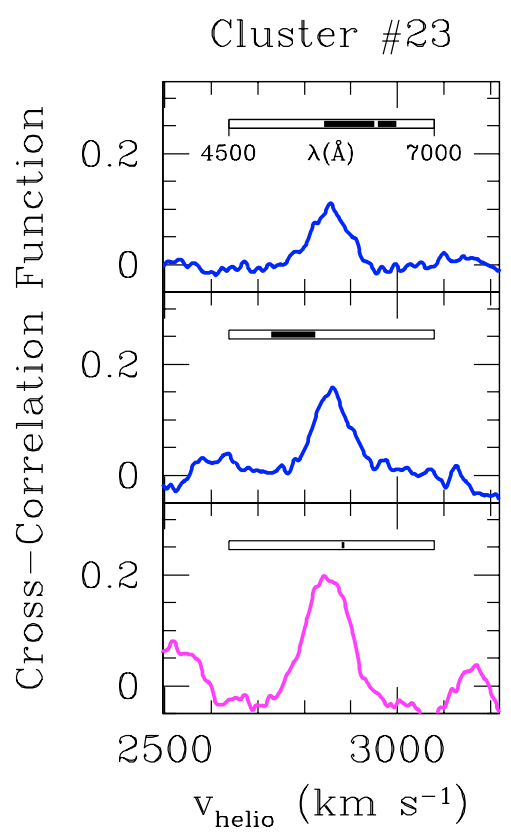

Fig. 6. Examples of cross-correlation functions of cluster \#23, here using the K4 supergiant HR 6961 as template star, in spectral regions R (top) and $\mathrm{G}_{R}$ (middle; see Table 3 for details) and in the region around the Na I D lines towards \#23 (bottom). The ranges in rest wavelength used for each cross-correlation are shown by the horizontal bar in each plot: the bar stretches from $4500 \AA$ to $7000 \AA$.

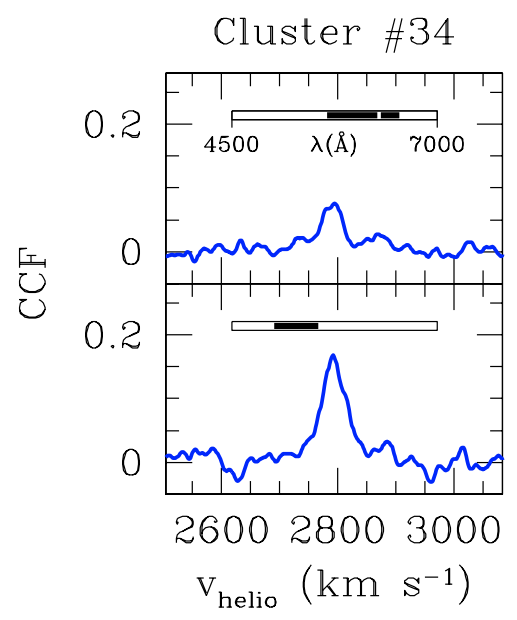

Fig. 7. Same as Fig. 6, but for cluster \#34 using the same template star. The upper panel shows spectral region $\mathrm{R}$, the lower panel $\mathrm{G}_{R}$.

\subsubsection{Calibrations and uncertainties}

For each cluster, spectral region and template star, we measured the heliocentric radial velocity $\left(v_{\text {helio }}\right)$, the amplitude $(h)$ and full width at half maximum $\left(W_{v}\right)$ of the best Gaussian fit to the peak of the cross-correlation function. To estimate $\Delta W$, the uncertainty in $W_{v}$, we made several measurements with fxcor of the width of each CCF peak, varying within plausible limits the baseline and velocity region for the fit, and taking the standard deviation of several measurements. Our notation is summarised in Table 4.

We measured $v_{\text {helio }}$ independently for each cluster and template star, using accurate radial velocities from the literature (Table 1). Since the catalogue velocity of HR 8299 was only accurate to $10 \mathrm{~km} \mathrm{~s}^{-1}$, and no catalogue velocity was available for 
Table 4. Notation for cross-correlation analysis.

\begin{tabular}{ll}
\hline \hline Symbol & Definition \\
\hline$v_{\text {helio }}$ & Heliocentric radial velocity \\
$W_{v}$ & Measured $F W H M$ of the CCF peak \\
$\Delta W$ & Estimated uncertainty of $W_{v}$ \\
$h$ & Peak value of the CCF \\
$\Delta h$ & Tolerance in $h$ matching \\
$\sigma$ & Velocity dispersion \\
$s_{W}$ & rms scatter in $W_{v}$ of derived $W_{v}-\sigma$ relation \\
$s_{\sigma}$ & rms scatter in $\sigma$ of derived $W_{v}-\sigma$ relation \\
\hline
\end{tabular}

HR 7706, we cross-correlated these with HR 6961 and measured new heliocentric velocities for these stars (Table 1).

To estimate the line-of-sight velocity dispersion $\sigma$ from width of the CCF, we followed the method of Ho \& Filippenko (1996a,b). The basic procedure was as follows: we first broadened each template star spectrum with Gaussians of known $\sigma$, which we then cross-correlated with the corresponding observed template star spectrum. From the resulting CCF, we measured the width $\left(W_{v}\right)$ and amplitude $(h)$ of the best Gaussian fit to the peak, in the same way as for the cluster spectra, and used this to calibrate a relation that gave the velocity dispersion $\sigma$ as a function of the measured CCF width $W_{v}$.

Our clusters are however faint, and our CCFs peak at lower values than those of previous authors. We therefore adapted the method in order to give a more careful calibration for our data.

To explore how the quality of the spectra affects the $W_{v}-\sigma$ calibration, we added different amounts of random, Gaussian noise to the template star spectra using the IRAF task mknoise. Each of these noisy model spectra was then cross-correlated with the corresponding observed template star spectrum. From among the resulting CCFs, we selected those whose peak amplitude $h$ matched that of the the observed cluster-template CCF, within a tolerance $\Delta h$ (Fig. 8), and used those data for our $W_{v}-\sigma$ calibration.

For CCF peak amplitudes comparable to those of our observations, we found that the relation between fitted CCF width $W_{v}$ and input $\sigma$ was always well-described by a linear relation whose parameters vary with template star and wavelength region. The value of the heliocentric velocity was not affected by low CCF peak amplitude, to within the errors.

To obtain a realistic estimate of the errors in the velocity dispersion calibration, we defined an acceptable spread $\Delta h$ about the measured CCF peak amplitude $\left(h_{\text {meas }}^{C} \pm \Delta h\right)$, and used only points lying in this interval. Here, $h_{\text {meas }}^{C}$ is the measured CCF peak amplitude for cluster $C$. The value of $\Delta h$ defines how closely we require the peak amplitude of our simulated CCFs to match that of the CCF peak from cross-correlating the cluster data with the template stars. The uncertainty in the derived value of $\sigma$ is clearly related to the scatter in each fit (see Fig. 8), but it also depends on the value of $\Delta h$. For example, when $\Delta h$ is large, the scatter ${ }^{6}$ about the linear relation between $W_{v}$ and $\sigma$ is also large.

For each combination of cluster, template star and spectral region, we adjusted the value of $\Delta h$ until $s_{W}$, the scatter in the CCF width, approached $\Delta W$, the uncertainty we estimated from using fxcor to measure the same CCF width (e.g. Fig. 8). In most cases, $s_{W}$ was somewhat larger than $\Delta W$, even for arbitrarily small values of $\Delta h$. Hence the uncertainty in the derived $\sigma$ values is not only due to uncertainty in

${ }^{6}$ We denote the RMS scatter in $W_{v}$ and $\sigma$ about the linear relation as $s_{W}$ and $s_{\sigma}$, respectively.

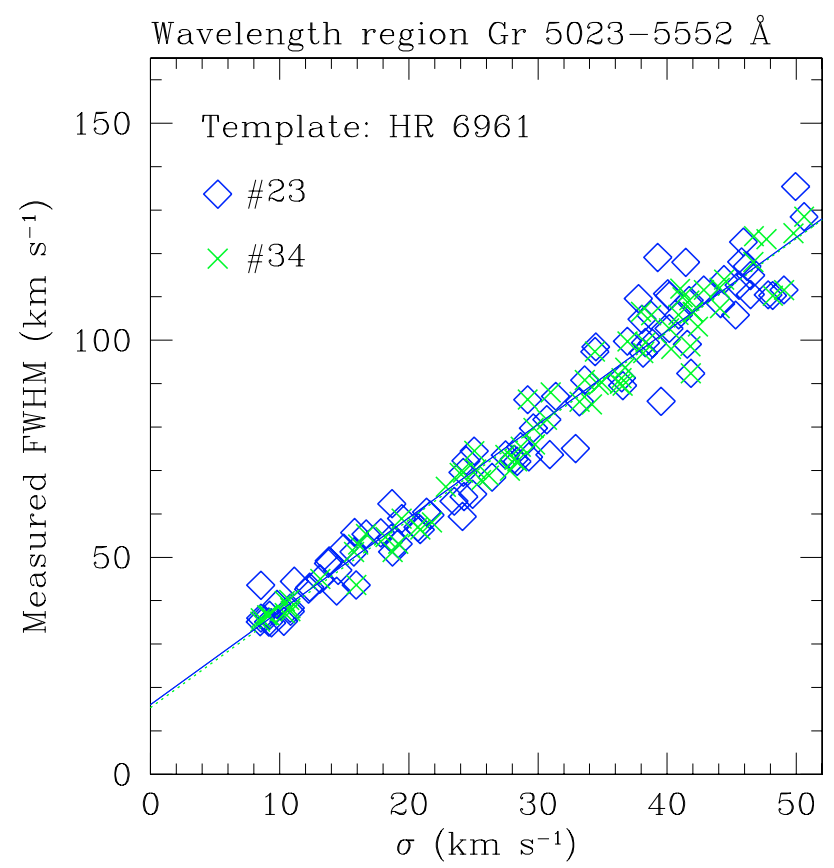

Fig. 8. An example plot showing how we have estimated velocity dispersion $\sigma$ by measuring the width of fits to the CCF peak. Diamonds represent models which match, to within \pm 0.015 , the observed CCF peak amplitude for cluster \#23. Crosses match the peak amplitude for \#34.

measuring $W_{v}$ but includes a small additional component from the cross-correlation method itself. Hence, $s_{\sigma}$ should give a realistic estimate of the uncertainty in each $\sigma$ measurement, and these are the values quoted in parentheses in Table 5.

The derived velocity dispersions show some variation with template star, though where the CCF amplitude is highest (region $\mathrm{G}_{R}$ ), the derived values of $\sigma$ are gratifyingly similar. The F5 V template star HR 7749 gives systematically lower values of $\sigma$ for both clusters, but only towards the blue, where we expect more contamination from early-type stars. For these reasons, and those outlined in Sect. 3.4.2, we regard the blue part of the $\mathrm{G}$ window as less reliable and prefer therefore the results from region $\mathrm{G}_{\mathrm{R}}$. The higher measured dispersion in $\mathrm{R}_{R}$ could be due in part to contamination from the broader lines seen in for example Si II in some early-type stars (UVESPOP database, Bagnulo et al. 2003). However, a velocity dispersion that varies as a function of wavelength is not necessarily unphysical but may arise naturally due to mass segregation, although we do not claim that this is the reason for the present discrepancy.

As we argued in Sect. 3.4.1, early-type supergiants are not likely contributors to the absorption spectra in the visual region of either \#23 or \#34. We nevertheless carried out some tests with an A-type supergiant as template star, in order to further constrain the dependence of our results on the assumed template star. The A2 Iab star HD 102878 from the UVESPOP database (Bagnulo et al. 2003) was used for this purpose. We found that while this star gave acceptable cross-correlation signals, indicating the same radial velocities as for our standard set of templates, we were unable to use it for obtaining a robust $\sigma-W_{v}$ calibration relation for our noisy data. Better results were obtained, however, for a hybrid template star which we constructed by adding HD 102878 and the K4 supergiant HR 6961 in proportions 1:4. This hybrid star produced radial velocities and $\sigma$ fully consistent with those of our normal template stars. Hence, even if 
Table 5. Derived velocity dispersions in $\mathrm{km} \mathrm{s}^{-1}$ for the two clusters, for each template star and spectral region. Errors are 1-sigma and are equal to the rms scatter in the $\sigma-W_{v}$ relation for each particular measurement; see text for details.

\begin{tabular}{|c|c|c|c|c|c|c|}
\hline \multirow{2}{*}{$\begin{array}{l}\text { ref. } \\
\text { star }\end{array}$} & \multirow{2}{*}{$\begin{array}{l}\text { Type/ } \\
\text { class }\end{array}$} & \multicolumn{5}{|c|}{ Spectral region (see Table 3) } \\
\hline & & $\mathrm{G}$ & $\mathrm{G}_{R}$ & $\mathrm{R}_{B}$ & $\mathrm{R}$ & $\mathrm{R}_{R}$ \\
\hline \multicolumn{7}{|c|}{ Cluster \#23 } \\
\hline HR 6961 & K4 Ib & $33.4(2.5)$ & $32.3(2.2)$ & $27.4(5.1)$ & $36.2(3.1)$ & $38.6(2.8)$ \\
\hline HR 7277 & K1 I & $33.0(3.2)$ & $32.8(2.4)$ & $29.7(6.7)$ & $37.2(2.9)$ & $48.6(7.8)$ \\
\hline HR 7706 & K1 III & $32.5(3.1)$ & $31.3(3.1)$ & $29.3(3.6)$ & $33.2(4.8)$ & $41.1(4.4)$ \\
\hline HR 7749 & F5 v & $21.2(2.7)$ & $32.0(2.4)$ & $30.9(3.5)$ & 39.7 (3.9) & $46.0(6.2)$ \\
\hline HR 8299 & G5 III & $35.4(2.1)$ & $34.6(3.3)$ & $39.5(6.1)$ & $37.7(2.4)$ & $43.8(4.6)$ \\
\hline Hybrid $^{1}$ & $\mathrm{~K}+\mathrm{A}$ & $31.9(3.4)$ & $29.9(3.5)$ & $29.4(5.0)$ & $30.2(2.4)$ & $37.5(13)$ \\
\hline \multicolumn{7}{|c|}{ Cluster \#34 } \\
\hline HR 6961 & K4 Ib & $18.6(2.4)$ & $17.4(2.0)$ & $19.1(2.8)$ & $15.8(3.0)$ & $34.3(19)$ \\
\hline HR 7277 & K1 I & $18.2(2.4)$ & $17.6(1.7)$ & $16.2(4.4)$ & $11.0(5.6)$ & $18.2(4.4)$ \\
\hline HR 7706 & K1 III & $18.0(2.5)$ & $16.8(3.0)$ & $16.3(3.5)$ & $15.8(3.5)$ & $15.8(8.0)$ \\
\hline HR 7749 & F5 V & $9.1(1.9)$ & $17.4(2.0)$ & $16.0(3.5)$ & $22.1(3.3)$ & $55.8(30)$ \\
\hline HR 8299 & G5 III & $18.8(1.7)$ & $18.1(1.8)$ & $17.6(8.2)$ & $18.1(2.7)$ & $20.3(32)$ \\
\hline Hybrid $^{1}$ & $\mathrm{~K}+\mathrm{A}$ & $18.5(2.3)$ & $18.4(2.4)$ & $15.6(5.2)$ & $15.3(2.4)$ & $18.5(15)$ \\
\hline
\end{tabular}

Note: (1) K4 Ib and A2 Iab in proportion 4:1.

Table 6. Derived heliocentric velocities in $\mathrm{km} \mathrm{s}^{-1}$ for the two clusters, for each template star and spectral region. Errors are formal errors in Gaussian fitting to the CCF peak from fxcor.

\begin{tabular}{|c|c|c|c|c|c|c|}
\hline \multirow{2}{*}{$\begin{array}{l}\text { ref. } \\
\text { star }\end{array}$} & \multirow{2}{*}{$\begin{array}{l}\text { Type/ } \\
\text { class }\end{array}$} & \multicolumn{5}{|c|}{ Spectral region (see Table 3) } \\
\hline & & G & $\mathrm{G}_{R}$ & $\mathrm{R}_{B}$ & $\mathrm{R}$ & $\mathrm{R}_{R}$ \\
\hline \multicolumn{7}{|c|}{ Cluster \#23 } \\
\hline HR 6961 & K4 Ib & $2860(7)$ & $2860(4)$ & $2858(7)$ & $2857(7)$ & $2855(7)$ \\
\hline HR 7277 & K1 I & $2857(7)$ & $2858(4)$ & $2855(6)$ & $2854(7)$ & $2853(7)$ \\
\hline HR 7706 & K1 III & $2860(8)$ & $2861(5)$ & $2857(9)$ & $2856(9)$ & $2856(9)$ \\
\hline HR 7749 & F5 V & $2858(8)$ & $2860(5)$ & $2857(8)$ & $2854(9)$ & $2853(8)$ \\
\hline HR 8299 & G5 III & $2859(7)$ & $2860(4)$ & $2857(7)$ & $2855(7)$ & $2854(7)$ \\
\hline Hybrid $^{1}$ & $\mathrm{~K}+\mathrm{A}$ & $2860(7)$ & $2859(4)$ & $2857(8)$ & $2857(7)$ & $2856(7)$ \\
\hline \multicolumn{7}{|c|}{ Cluster \#34 } \\
\hline HR 6961 & $\mathrm{~K} 4 \mathrm{Ib}$ & $2794(4)$ & $2795(2)$ & $2793(5)$ & $2792(5)$ & $2789(14)$ \\
\hline HR 7277 & $\mathrm{~K} 1 \mathrm{I}$ & $2792(4)$ & $2793(2)$ & $2791(5)$ & $2790(5)$ & $2789(5)$ \\
\hline HR 7706 & K1 III & $2794(4)$ & $2796(2)$ & $2793(7)$ & $2793(5)$ & $2793(5)$ \\
\hline HR 7749 & F5 V & $2793(4)$ & $2794(2)$ & $2793(7)$ & $2790(8)$ & $2785(13)$ \\
\hline HR 8299 & G5 III & $2794(3)$ & $2795(2)$ & $2793(6)$ & $2793(6)$ & $2792(5)$ \\
\hline Hybrid $^{1}$ & $\mathrm{~K}+\mathrm{A}$ & $2794(4)$ & $2795(2)$ & $2792(5)$ & $2792(5)$ & $2791(5)$ \\
\hline
\end{tabular}

Note: (1) K4 Ib and A2 Iab in proportion 4:1.

early-type stars could contribute to the continua, they would not contribute much to the CCF signal.

\subsubsection{Results for cluster \#23}

In Tables 5 and 6 we present the resulting velocity dispersions and radial velocities for the different wavelength regions and template stars.

For \#23, we find a weighted average value of $\sigma_{23}=32.5 \pm$ $2.7 \mathrm{~km} \mathrm{~s}^{-1}$ in region $\mathrm{G}_{R}$ (Sect. 3.4.2), when using all 5 template stars, and $\sigma_{23}=32.5 \pm 2.3$ if we only use the two $\mathrm{K}$ supergiants (see discussion in Sect. 3.4.1). The uncertainty here includes both the uncertainty in each measurement $\left(s_{\sigma}\right.$; the values quoted in parentheses in Table 5, see Sect. 3.4.3), and the smaller variation between results for different template stars (i.e. the standard deviation of the $\sigma$ column in question). We use this method to estimate the total uncertainty in $\sigma$ in what follows. For region $\mathrm{R}$ we find $\sigma_{23}=37.1 \pm 3.4 \mathrm{~km} \mathrm{~s}^{-1}$ when using all stars and $\sigma_{23}=36.7 \pm 3.1$ when using the $\mathrm{K}$ supergiants only. Combining the measurements from $\mathrm{G}_{R}$ and $\mathrm{R}$ we obtain $\sigma_{23}=34.4 \pm 3.8 \mathrm{~km} \mathrm{~s}^{-1}$ when using all stars, and $\sigma_{23}=34.1 \pm 3.5 \mathrm{~km} \mathrm{~s}^{-1}$ when using only the K supergiants.

Following the discussion in Sect. 3.4.1 giving higher weight to the $\mathrm{K}$ super giants, and noting the better quality of the $\mathrm{G}_{R}$ data, we adopt as our best estimate $\sigma_{23}=32.5 \pm 2.5 \mathrm{~km} \mathrm{~s}^{-1}$. We note that adopting a velocity dispersion of the magnitude implied from region $\mathrm{R}$ would produce a virial mass that is $25 \pm 25 \%$ higher.

We find heliocentric velocities $v_{\text {helio,23 }}=2859.7 \mathrm{~km} \mathrm{~s}^{-1}$ in $\mathrm{G}_{R}$ and 2855.2 in $\mathrm{R}$, with a scatter between the five template stars that in both cases is only $\sim 1 \mathrm{~km} \mathrm{~s}^{-1}$. Restricting to $\mathrm{K}$ supergiants only makes no significant difference for $v_{\text {helio,23. }}$. The output uncertainties on $v_{\text {helio }}$ from fxcor are on the order of $4 \mathrm{~km} \mathrm{~s}^{-1}$ in $\mathrm{G}_{R}$ and $8 \mathrm{~km} \mathrm{~s}^{-1}$ in $\mathrm{R}$ (see Table 6). The small star-to-star scatter suggests that the uncertainty is dominated by the quality of our cluster spectra rather than by template star mismatches. We note a systematic difference of $\sim 4 \mathrm{~km} \mathrm{~s}^{-1}$ between regions $\mathrm{G}_{R}$ and $\mathrm{R}$, that however is within the uncertainties. We base our final value on the $\mathrm{G}_{R}$ data: $v_{\text {helio,23 }}=$ $2859.7 \pm 4.4 \mathrm{~km} \mathrm{~s}^{-1}$. 
Table 7. Properties of clusters 23 and 34. The colours, absolute magnitudes and mass-to-light ratios are corrected for Galactic reddening according to Schlegel et al. (1998), but not for internal reddening.

\begin{tabular}{lcc}
\hline \hline Cluster no. & $\# 23$ & $\# 34$ \\
\hline Absolute magnitude $M_{v}$ & -15.5 & -12.8 \\
Colour $(v-i)$ & 0.11 & 0.86 \\
Effective radius $r_{\mathrm{e}}(\mathrm{pc})$ & $5.2 \pm 1.0$ & $5.6 \pm 0.4$ \\
Heliocentric velocity $v_{\text {helio }}\left(\mathrm{km} \mathrm{s}^{-1}\right)$ & $2859.7 \pm 4.4$ & $2794.6 \pm 2.2$ \\
Velocity dispersion $\sigma\left(\mathrm{km} \mathrm{s}^{-1}\right)$ & $32.5 \pm 2.5$ & $17.6 \pm 2$ \\
Virial mass $\left(10^{6} M_{\odot}\right)$ & $13 \pm 3$ & $4.0 \pm 1$ \\
Mass-to-light ratio $\left(M / L_{V}\right)_{\odot}$ & $0.093 \pm 0.02$ & $0.35 \pm 0.09$ \\
\hline
\end{tabular}

To investigate possible contamination from other clusters, we compared cross-correlation results for the 1.8-arcsec extraction with the narrower, 1-arcsec wide, extraction around cluster \#23 (see Sect. 2.2). The narrower extraction gives CCFs with poorer signal-to-noise ratio but very similar velocity dispersion in both $\mathrm{G}_{R}$ and $\mathrm{R}$. Also, the use of hybrid templates made from HD 102878 (A supergiant) and HR 6961 (K supergiant) produced very similar results $\left(\sigma_{23}=30 \mathrm{~km} \mathrm{~s}^{-1}\right.$ in both $\mathrm{G}_{R}$ and $\left.\mathrm{R}\right)$.

\subsubsection{Results for cluster \#34}

For cluster \#34 (Tables 5 and 6) the analysis was more straightforward. We measured $\sigma_{34}=17.6 \pm 2.0 \mathrm{~km} \mathrm{~s}^{-1}$ in $\mathrm{G}_{R}$ and $17.4 \pm$ 4.4, in R. Combining $\mathrm{G}_{R}$ and $\mathrm{R}$ yields $\sigma_{34}=17.5 \pm 2.8 \mathrm{~km} \mathrm{~s}^{-1}$.

For the radial velocities we find $v_{\text {helio,34 }}=2794.6 \pm 2.2 \mathrm{~km} \mathrm{~s}^{-1}$ in $\mathrm{G}_{R}$ and $2791.7 \pm 5.6$ in $\mathrm{R}$, i.e. we see a similar systematic difference between $\mathrm{G}_{R}$ and $\mathrm{R}$ as for cluster \#23, but which again is within the uncertainties.

Given the better quality of the $\mathrm{G}_{R}$ data, we adopt these results for $\sigma_{34}$ and $v_{\text {helio,34. }}$

\subsection{Evidence for ISM gas flows from Na I lines}

We also carried out cross-correlations on the narrow spectral region 5874-5912 $\AA$, which is dominated by the Na I D doublet. For cluster \#23 this was successful (see Fig. 6), but the wavelength region was too short to yield anything useful in the more noisy spectrum of \#34. Nor could we obtain a reliable velocity dispersion calibration. The result would in any case be difficult to interpret, since we might also expect a contribution from ISM absorption. The heliocentric velocity is well-determined and remarkably low. At $2845 \pm 7 \mathrm{~km} \mathrm{~s}^{-1}$ it is $25 \mathrm{~km} \mathrm{~s}^{-1}$ lower than that inferred from region $\mathrm{G}_{R}$ along the same line of sight. Presumably, the Na I absorption we are seeing here is a combination of both stellar lines and cold interstellar medium moving at a speed of $\sim 20 \mathrm{~km} \mathrm{~s}^{-1}$ along the line of sight to the cluster (cf. Schwartz \& Martin 2004). The emission lines also show evidence for a blue-shifted component (see Sect. 3.8).

\subsection{Cluster sizes}

We have measured the spatial extent of the two clusters using the HST images described and presented by Östlin et al. (1998).

To measure the sizes of \#23 and \#34 we used curves of growth extending from 0 to 5 Planetary Camera (PC) pixels. One PC pixel has a size of $0{ }^{\prime} 0455$ which corresponds to $8.27 \mathrm{pc}$ at the adopted distance of ESO 338-IG04. We used observations in 4 passbands: $F 336 W, F 439 W, F 555 W$ and $F 814 W$.
The growth curves were compared to model PSFs generated with Tiny $\operatorname{Tim}^{7}$ (Krist \& Hook 2003) that we convolved with Gaussian profiles and King profiles with a concentration parameter $c=2$.

Using the whole growth curve from 0 to 5 pixels, the resulting sizes are well-constrained. We find the best-fitting effective half-light radii to be $r_{\mathrm{e}}=5.2 \pm 1.0$ and $5.6 \pm 0.4 \mathrm{pc}$ for \#23 and \#34, respectively, when using a Gaussian profile. Since the difference in estimated effective radius is in general small when comparing Gaussians and different King model assumptions, $r_{\mathrm{e}}$ is a quite model-independent, and hence robust, measure of the size of a cluster (Carlson \& Holtzman 2001; Maraston et al. 2004).

\subsection{Virial masses}

From the virial theorem, we can estimate the mass of a cluster if we know its velocity dispersion $\sigma$ and its size. Assuming the cluster is in virial equilibrium, its mass is given by

$M_{\text {vir }}=k \sigma^{2} r_{\mathrm{m}} / G$,

(e.g. Spitzer 1987; Smith \& Gallagher 2001; McCrady et al. 2003) where $r_{\mathrm{m}}$ is the half-mass radius. Taking $k=7.5, r_{\mathrm{m}}=\frac{4}{3} r_{\mathrm{e}}$ (Spitzer 1987), we find that the cluster masses are given by:

$M_{\mathrm{vir}}=2.324 \times 10^{3}\left(\frac{\sigma}{\mathrm{km} \mathrm{s}^{-1}}\right)^{2}\left(\frac{r_{\mathrm{e}}}{\mathrm{pc}}\right) M_{\odot}$

Specifically, for \#23,

$M_{23}=1.28 \times 10^{7}\left(\frac{\sigma_{23}}{32.5 \mathrm{~km} \mathrm{~s}^{-1}}\right)^{2}\left(\frac{r_{\mathrm{e}}}{5.2 \mathrm{pc}}\right) M_{\odot}$

and for \#34,

$M_{34}=4.03 \times 10^{6}\left(\frac{\sigma_{34}}{17.6 \mathrm{~km} \mathrm{~s}^{-1}}\right)^{2}\left(\frac{r_{\mathrm{e}}}{5.6 \mathrm{pc}}\right) M_{\odot}$.

In Table 7 we summarise the properties of clusters \#23 and \#34, including the resulting dynamical mass-to-light ratios $\left(M / L_{V}\right)$. The uncertainty intervals follows from a root-square addition of the uncertainties in $\sigma$ and $r_{\mathrm{e}}$. Boily et al. (2005) discuss the evolution of $\eta=k \cdot r_{\mathrm{m}} / r_{\mathrm{e}}$ with time during the evolution of a cluster. For a cluster of the age of \#23, i.e. $\sim 5 \mathrm{Myr}$, Boily et al. (2005) find $\eta \approx 10$, which is what we have used above. For ages greater than $50 \mathrm{Myr}$, however, they predict $\eta \approx 20$ for models with high initial density.

\footnotetext{
${ }^{7}$ http://www.stsci.edu/software/tinytim
} 


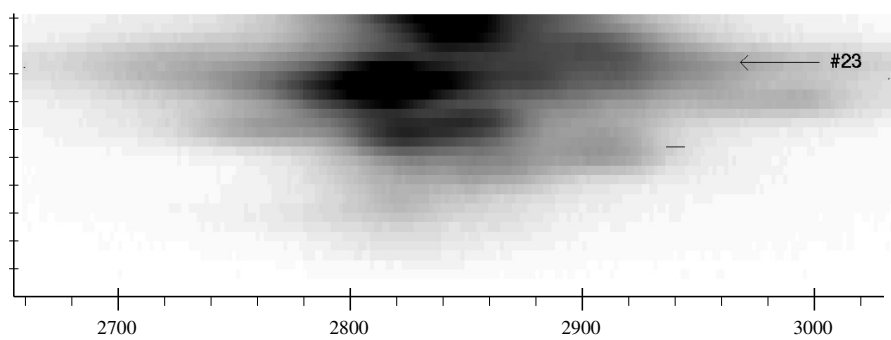

Fig. 9. Two-dimensional spectrum around [O III] $\lambda 5007$ showing the complex velocity structure in the ionised gas. The $x$-axis is labelled with velocity in units of $\mathrm{km} \mathrm{s}^{-1}$. The $y$-axis is the location along the slit, and the tick marks have a spacing of one arcsecond. The position of \#23 is indicated by an arrow, and two high velocity structures are marked by short dashes.

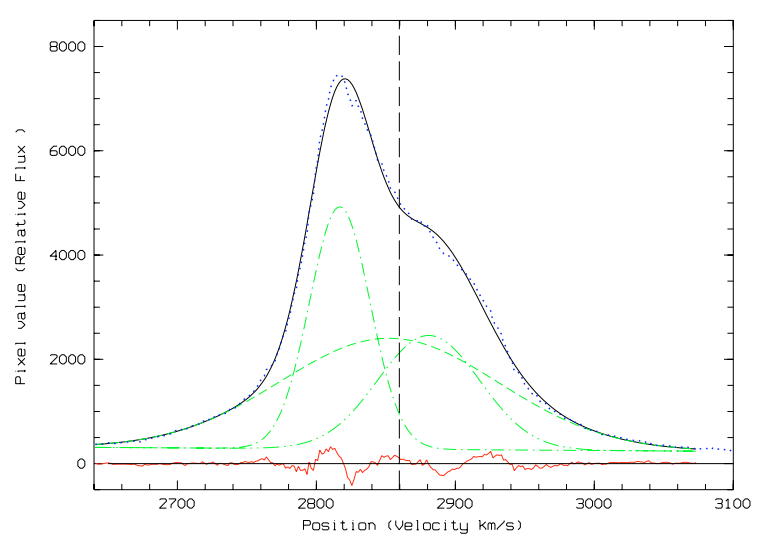

Fig. 10. $\mathrm{H} \alpha$ line profile for the two scan lines centred on \#23, without background subtraction. The $x$-axis is labelled in velocity. The thin (black) solid line shows the observed spectrum. The (blue) dotted line shows the modelled spectrum which here is a sum of 3 Gaussian profiles, which are shown as (green) dashed, dash-dot and dash-dot-dot lines. The (red) dotted line at the bottom shows the residuals (observed - model spectrum). The vertical (black) long-dashed line shows the velocity of \#23 determined from absorption lines through crosscorrelation. This spectrum shows both that there is both a blueshifted and a redshifted component, and that there is a broad component with velocity close to the velocity of \#23.

\subsection{Velocity structure in emission lines}

The slit covers a region of strong emission from ionised gas. In Fig. 9 we show a spectral image centred on [O III] $\lambda 5007$ showing that the ionised gas motions along the slit are complex. The same features are seen in $\mathrm{H} \alpha$. The wavelength of the peak of the $\mathrm{H} \alpha$ and [O III] $\lambda 5007$ lines varies by almost $100 \mathrm{~km} \mathrm{~s}^{-1}$ along the slit, but not in a monotonic fashion. Closer inspection reveals the presence of several velocity components at each position along the slit. For most positions at least three Gaussians appear to be needed to adequately reproduce the observed emission.

Figure 10 shows the $\mathrm{H} \alpha$ line profile at the location of \#23. The line is very broad and clearly non-Gaussian, with peak intensity blue-shifted by $45 \mathrm{~km} \mathrm{~s}^{-1}$ with respect to the velocity inferred from the absorption lines in \#23. We fit three Gaussians to the observed profile and find, one narrow blueshifted component (at $-40 \mathrm{~km} \mathrm{~s}^{-1}$ with respect to the absorption line velocity), one redshifted component (at $+20 \mathrm{~km} \mathrm{~s}^{-1}$ ), and one broad $\left(\sigma=65 \mathrm{~km} \mathrm{~s}^{-1}\right)$ component with nearly the same velocity as \#23. We see the same pattern in both H $\alpha$ and [O III] $5007 \AA$.

Looking at the scan lines to the north-east of cluster \#23 (above \#23 in Fig. 9), the line is split into two components: one blueshifted and one redshifted with respect to the centre of the cluster. For the scan lines immediately below cluster \#23, the blueshifted component dominates.

The blue- and red-shifted components suggest a bubble expanding with a velocity of $\sim 40 \mathrm{~km} \mathrm{~s}^{-1}$. If we take a characteristic radius of $1^{\prime \prime}$ (see Fig. 9 and Sect. 4.1), the timescale for blowing such a bubble is $\sim 5 \mathrm{Myr}$, which is of the same order as the age of \#23. Our analysis here also shows that emission lines, being much broader than the cluster's absorption lines, cannot reliably be used to infer the dynamical mass of associated clusters (cf. Turner et al. 2003). A few scan lines further down (marked with ticks in Fig. 9) we see high-velocity components, redshifted by $200 \mathrm{~km} \mathrm{~s}^{-1}$ and $80 \mathrm{~km} \mathrm{~s}^{-1}$. Finally we note that cluster \#34 has a stellar absorption line velocity which is $65 \mathrm{~km} \mathrm{~s}^{-1}$ less than the ionised gas from the same position on the slit.

\section{Discussion: Comparing cluster masses and ages from photometry and spectroscopy}

The ages and photometric masses of the cluster population in ESO 338-IG04 have been investigated by Östlin et al. (2003). Here, we discuss the properties of cluster \#23 and \#34 in more detail, and compare with the virial mass estimates and age estimates based on our UVES spectra.

For the sub-population of young (age $\leq 20 \mathrm{Myr}$ ) clusters, Östlin et al. (2003) preferred a low metallicity for both the stellar and ionised gas components. This agrees with the nebular oxygen abundance of $12+\log (\mathrm{O} / \mathrm{H})=8.0$ (Bergvall \& Östlin 2002). Moreover, to reproduce the youngest clusters, the IMF needs to have a slope equal to or flatter than the Salpeter value ( $\alpha=2.35)$ and an upper mass limit $M_{\text {up }} \geq 60 M_{\odot}$. We shall refer to this preferred metal-poor model as the "standard" $\operatorname{model}^{8}$ in the following sections.

In the absence of dark matter, the photometric and dynamical masses should agree. If this is not the case, the reason may be sought either in the determination of the dynamical mass (because of mass segregation, or non-equilibrium), or the photometric mass (IMF, stellar parameters). Below we discuss the photometric and dynamical masses of each cluster in this context.

\subsection{Cluster \#23}

The standard model gives an age of $7 \mathrm{Myr}$, an internal reddening of $E_{B-V}=0.05$ and a photometric mass of $5 \times 10^{6} M_{\odot}$, i.e. significantly lower than the virial mass. By adopting a steeper IMF slope $(\alpha=2.85)$ or a low upper mass limit $\left(M_{\text {up }}=20 M_{\odot}\right)$, photometric masses in the range 2 to $6 \times 10^{7} M_{\odot}$ can be produced.

The models of Zackrisson et al. $(2001 ; \mathrm{Z} 01)$ that were used in Östlin et al. (2003) include nebular emission. However, the nebular emission produced by each cluster is assumed to be included in the photometric aperture, which has a radius of $0 .{ }^{\prime} 14$, or $25 \mathrm{pc}$ at the distance of ESO 338-IG04. This assumption appears to be valid for the majority of young clusters, but it does not hold for \#23. This cluster alone accounts for just over $10 \%$ of the integrated starburst flux at $2000 \AA$ and has ionised a large bubble (radius $\sim 1^{\prime \prime}$, i.e. nearly $200 \mathrm{pc}$ ), which, as we discussed in Sect. 3.8, is expanding (Figs. 9 and 10; see also Fig. 2 in Östlin et al. 2003, or Fig. 7 in Hayes et al. 2005). Since ionised gas dominates the luminosity output when the cluster is young

\footnotetext{
8 Standard model: instant burst, IMF with slope $\alpha=2.35$ (Salpeter) and mass range $0.08-120 M_{\odot}$, gas covering factor of unity, metallicity of stars and gas: $Z_{\text {gas }}=0.002, Z_{\text {stars }}=0.001$.
} 
(Bergvall \& Östlin 2002), the total luminosity, and hence the photometric mass of \#23, may be underestimated by at most a factor of about two (see Fig. 17 in Bergvall \& Östlin 2002). For instantaneous bursts of age $\sim 6 \mathrm{Myr}$, the correction to the mass is instead of the order $+25 \%$. The displaced nebular emission also means that the results of the photometric modelling for \#23 are more uncertain than for most other young clusters in ESO 338-IG04.

Indeed, recent $\mathrm{H} \alpha$ photometry with HST/ACS (Östlin et al., in prep.), gives an integrated $\mathrm{H} \alpha$ luminosity for \#23 (within the same aperture as above) of $\sim 10^{32} \mathrm{~W}$. For the electron density derived from our UVES spectra (Sect. 3.1), this implies a total ionised gas mass of $\sim 10^{4} M_{\odot}$ or about one thousandth of the dynamical mass. We caution that the density is derived from a larger region where the nebular component is dominated by the more extended gas around \#23, but the value is sufficiently small that we can conclude that the mass of ionised gas in \#23 is negligible compared to the dynamical and stellar masses. Moreover, the $\mathrm{H} \alpha$ emission line equivalent width for \#23 derived from the ACS images is only about $\sim 50 \AA$, much smaller than predicted for an age of $\sim 7$ Myr (Starburst99, Leitherer et al. 1999). Hence, the ionising photons produced in \#23 leak out over a much larger volume than the size of the cluster. Much of the ISM originally present in this cluster is likely to have been removed already.

Another reason for the discrepant masses could be that the cluster is not in virial equilibrium. This could be the case if the cluster is so young that it has not yet had time to virialise, or because supernova explosions have led to the prompt expulsion of gas from the cluster. In such cases its dynamical mass may be overestimated by a factor of up to 3, depending on the star formation efficiency (SFE) in the cluster, i.e. the mass fraction of gas initially present that was turned into stars (Bastian \& Goodwin 2006). In the case of $\# 23$ we would infer a SFE of $\sim 40 \%$ for a stellar mass of $5 \times 10^{6} M_{\odot}$. If we account for the displaced nebular emission, we obtain SFE $>50 \%$, the exact amount depending on the dynamical state of the cluster prior to gas expulsion. If the fraction of gas lost is larger than $70 \%$ the cluster may dissolve completely, but even a cluster which remains bound will lose a few tenths of its initial stellar mass (Bastian \& Goodwin 2006). Hence we expect \#23 to remain bound but to lose a few tenths of its mass over the next $\sim 50 \mathrm{Myr}$.

Alternative constraints on the age and IMF can be obtained from the spectra presented in this paper. Our analysis of Balmer and He I absorption lines in Sect. 3.3 indicated an age of $6_{-2}^{+4}$ Myr. The effect of adopting an IMF with relatively few massive stars is to increase the equivalent widths at young ages. A low upper mass limit $\left(M_{\text {up }} \geq 30 M_{\odot}\right)$ may still be marginally consistent with the data, but an IMF slope significantly steeper than the Salpeter value predicts equivalent widths that are too high (see for example Fig. 11 in González Delgado et al. 1999).

More evidence for the the presence of very massive stars comes from the detection of intermediate-width stellar He II $\lambda 4686$ line emission with equivalent width $0.8 \AA$. We see no obvious WR features but interpret this line as a signature of Of stars, which are very massive stars that may be on their way to becoming WR stars (Nota et al. 1996; Schaerer \& Vacca 1998). This observation makes an IMF with a low upper mass limit improbable, but is consistent with a Salpeter IMF and an age $\leq 5$ Myr (Schaerer \& Vacca 1998; Leitherer et al. 1999).

We noted in Sect. 3.4.1 that the presence of strong calcium triplet absorption from \#23 signals the presence of red supergiants. For a Salpeter IMF and low metallicity, the presence of RSGs indicates an age of $\geq 5 \mathrm{Myr}$, whereas for an IMF with a low upper mass limit, the RSGs require that the cluster is older than 10 Myr. The simultaneous presence of RSGs and emission in He II $\lambda 4686$ is somewhat unexpected, but could be accounted for if the formation of the cluster extended over a few Myr, rather than being instantaneous. Similar scenarios have been presented for R136 (Massey \& Hunter 1998) and NGC 3603 (Pandey et al. 2000). Moreover, recent stellar models which include rotation predict longer WR lifetimes (Meynet \& Maeder 2005).

Fellhauer \& Kroupa (2005) have proposed that the anomalously massive, intermediate-age cluster W3 in NGC 7252 may be the product of merging of several less massive clusters. Though the timescale for this to happen seems short in the case of \#23, an earlier merger of young clusters could lead to the presence of stellar populations with different ages, and explain the simultaneous presence of Of stars and RSGs.

It is also quite possible that stellar evolution in a cluster as massive as \#23 is different compared to a lower-density environment. Numerical simulations of massive star clusters indicate that dynamical friction rapidly leads to strong mass segregation and possibly merging of the most massive stars (e.g. Portegies Zwart et al. 1999). Massive-star mergers would likely have a sizeable impact on the early spectral evolution of such a star cluster.

In conclusion, we find strong support for an IMF of \#23 that extends to high masses (on the order of $100 M_{\odot}$ ) and has a slope not significantly steeper than the Salpeter value. The cluster's photometric and dynamical masses are in reasonable agreement, when allowing for the fact that most of its associated nebular emission lies outside the photometric aperture, and the possibility that the cluster is not yet fully virialised. A more exotic possibility would be that the cluster contains of the order of $50 \%$ dark matter.

\subsection{Cluster \#34}

Cluster \#34 is much older than both \#23 and the current starburst. Our standard ${ }^{8}$ metal-poor model (considered in Östlin et al. 2003, based on the code by Zackrisson et al. 2001; Z01), implies an age of $\sim 1.5 \mathrm{Gyr}$, and photometric mass $2 \times 10^{7} M_{\odot}$, almost an order of magnitude larger than the virial estimate $\sim 4 \times$ $10^{6} M_{\odot}$. Compared to the photometry of \#34, this model is discordant at the 1-sigma level. Varying the IMF slope $\alpha$ in the range 1.35 to 2.85 gives comparable qualities of fit and a mass $>10^{7} M_{\odot}$. A better fit can be obtained by adopting a model with significantly higher metallicity ( $Z=0.008$ to 0.04 ). This results in a lower age, $250 \mathrm{Myr}$ (for $Z=0.04$ ) to $500 \mathrm{Myr}$ $(Z=0.008)$, and a somewhat lower mass of $\sim 10^{7} M_{\odot}$. For all assumed metallicities and IMF parameters, we find a rather high internal reddening, $E_{B-V} \approx 0.25$, which can be compared to the low values for the galaxy's starburst centre $\left(E_{B-V} \leq 0.05\right.$; Östlin et al. 2003) suggesting that \#34 may lie on the far side of ESO 338-IG04. About one third of the other old and intermediate age clusters have similar implied reddenings.

In addition to Z01, whose stellar evolutionary tracks come mainly from the Geneva group, we have used the spectral synthesis code PÉGASE. 2 (Fioc \& Rocca-Volmerange 1997, 1999) which is mainly based on tracks from the Padova group. One effect of adopting a different set of tracks is that PÉGASE. 2 in general implies a lower reddening and higher age for \#34. The best fit is again obtained for a rather metal-rich $(Z=0.008)$ model that gives an age of $1.2 \mathrm{Gyr}$, a reddening of $E_{B-V}=0.1$ and mass $1.1 \times 10^{7} M_{\odot}$ for a Salpeter IMF. However, PÉGASE. 2 gives results that are consistent with the photometry for all metallicities in the range $0.002<Z<0.05$, with resulting ages from 0.8 to $1.2 \mathrm{Gyr}$, and masses which differ by less than $20 \%$. 
In summary, models based on a Salpeter IMF predict a mass of $\sim 10^{7} M_{\odot}$, irrespective of the set of stellar evolutionary tracks or metallicity used.

All the mass estimates so far in this subsection assume a single power law IMF, including remnants but excluding the gas returned in the process of stellar evolution, which is assumed to have left the cluster. If we instead assume an IMF like that seen in the solar neighbourhood, with a flatter slope for stars with mass smaller than $0.5-1 M_{\odot}$, the photometric masses quoted above are reduced by a factor of $\sim 2$. For instance, a Scalo98 (Scalo 1998) IMF yields $\sim 5 \times 10^{6} M_{\odot}$ for the best fitting models. This is only slightly larger than the virial mass and such an IMF is also consistent with Galactic GCs (Chabrier \& Méra 1997).

The work by Boily et al. (2005) suggests that for ages larger than $50 \mathrm{Myr}$, masses may be underestimated by a factor of two if the temporal evolution of $\eta$ is not accounted for (see also Sect. 3.7). However, this is only true for clusters with high initial mass surface density, and moreover, the evolution past $50 \mathrm{Myr}$ is not known. Our \#34 has a present mass surface density of $\sim 2 \times 10^{4} M_{\odot} / \mathrm{pc}^{2}$ (without any $\eta$-evolution included), so strong effects are not expected.

Given the concordance of the photometric mass for different models and metallicities, we conclude that the dynamical mass of \#34 is best explained with an IMF where the low-mass part is similar to that observed in the solar neighbourhood and in Galactic GCs.

\subsection{Implications for the globular cluster system in ESO 338-IG04}

The two clusters studied in this paper are consistent with a normal IMF, extending to low stellar masses $\left(M \sim 0.1 M_{\odot}\right)$ and with a slope in the high mass regime $\left(M>1 M_{\odot}\right)$ close to the Salpeter value. Such IMFs will favour survival against cluster destruction mechanisms related to stellar death and gas expulsion. A few SSCs in other galaxies on the other hand seem to have rather odd IMFs, e.g. M82-F, for which a severe deficiency of low mass stars is implied (Smith \& Gallagher 2001), making its long-term survival unlikely.

All SSCs and GCs clusters line up nicely in a fundamental plane (Walcher et al. 2005) suggesting that initial conditions regulate their properties (McLaughlin 2000). That young SSCs, including \#23 and \#34, follow the same trends suggests that the same basic formation mechanism applies both for globular clusters in ancient halos and for present-day mergers. Östlin et al. (2003) used the cluster age distribution in ESO 338-IG04 to map its past star and cluster formation history, and found that star clusters seem to make up as much as several percent of the total stellar mass, in contrast to the value of $0.3 \%$ found for most galaxies (McLaughlin 2000).

Our study demonstrates the massive nature of at least some of the star clusters in ESO 338-IG04. More velocity dispersion measurements in ESO 338-IG04 would be illuminating. Though they are the brightest of the subpopulations of young and intermediate-age clusters, respectively, the objects studied in this paper are just two of the hundreds of clusters detected in this galaxy. Despite their impressive masses, \#23 and \#34 are apparently faint, with $m_{v}=17.4$ and 20.1, respectively. Cluster \#34 is the faintest so far for which velocity dispersions have been derived from optical spectroscopy. With the exception of two very massive intermediate age clusters in NGC 7252 (Bastian et al. 2006), our clusters are also the most distant yet probed. In this paper we have shown that velocity dispersion measurements of such faint clusters are indeed feasible, and we expect that clusters one magnitude fainter would be within reach with modest increase of integration time on VLT/UVES. Several more clusters in ESO 338-IG04 could be weighed by the same method.

\section{Conclusions}

We have presented high resolution spectra of two luminous star clusters in the luminous blue compact galaxy ESO 338-IG04 (Tololo 1924-416). The spectra have been cross-correlated with template stars observed using the same setup to determine each cluster's line-of-sight velocity dispersion. Using size estimates from Hubble Space Telescope images, we have used the velocity dispersions to determine the virial masses of the clusters. Our mass estimates have been compared to masses derived from HST photometry fitted to spectral evolutionary synthesis models. This comparison indicates that both clusters have rather normal IMFs, favouring their survival against internal disruption mechanisms.

One of the clusters (\#23) is young, with an age of 6-7 Myr and mass in excess of $10^{7} M_{\odot}$, making it one of the most massive very young clusters known. We find evidence for the simultaneous presence of massive $\mathrm{O}$-stars and red supergiants. The [O III] $\lambda 5007$ and $\mathrm{H} \alpha$ emission line profiles from the region surrounding cluster \#23 indicate the presence of a bubble expanding at a velocity $\sim 40 \mathrm{~km} \mathrm{~s}^{-1}$. In addition, we see signs of neutral gas flows along the line of sight from the cross-correlation analysis of the Na I D absorption lines. The IMF of this cluster shows no evidence for any deficiency of low-mass stars, but is consistent with a Salpeter slope over the whole mass range $0.08-120 M_{\odot}$. The inferred dynamical mass is larger than expected based on the luminosity of the cluster, which may indicate that the cluster is not yet virialised.

The other cluster (\#34) is older and has a mass of $4 \times 10^{6} \mathrm{M}_{\odot}$. A combined analysis with the photometric data suggests that it has an age of between 0.3 and 1.4 Gyr. The slope of the IMF at masses less than $1 M_{\odot}$ must be flatter than the Salpeter value for the virial and photometric masses to be consistent. Such an IMF is in agreement with that observed for old Galactic globular clusters. This work confirms that this cluster is a bona-fide young globular cluster.

Acknowledgements. We thank Andrea Modigliani for useful suggestions on the reduction of UVES data. The authors acknowledge financial support from the Swedish research council and the Swedish National Space Board. We thank Matthew Hayes, Erik Zackrisson, Kambiz Fathi and Jan-Olov Persson for useful discussions.

\section{References}

Arp, H., \& Sandage, A. 1985, AJ, 90, 1163

Bagnulo, S., Jehin, E., Ledoux, C., et al. 2003, Messenger, 114, 10

Bastian, N., \& Goodwin, S. 2006, MNRAS, 369, 9

Bastian, N., Saglia, R. P., Goudfrooij, P., et al. 2006, A\&A, 448, 881

Bergvall, N., \& Östlin, G. 2002, A\&A, 390, 891

Bertelli, G., Bressan, A., Chiosi, C., Fagotto, F., \& Nasi, E. 1994, A\&AS, 106, 275

Bica, E., \& Alloin, D. 1986, A\&A, 162, 21

Bruzual, G., \& Charlot, S. 2003, MNRAS, 344, 1000

Boily, C. M., Lancon, A., Dieters, S., \& Heggie, D. C. 2005, ApJ, 620, L27

Carlson, M. N., \& Holtzman, J. A. 2001, PASP, 113, 1522

Chabrier, G., \& Méra, D. 1997, A\&A, 328, 83

Díaz, A. I., Terlevich, E., \& Terlevich, R. 1989, MNRAS, 239, 325

Drilling, J. S., \& Landolt, A. U. 2000, in ed. A. N. Cox, Allen's Astrophysical Quantities, 4th edn., Am. Inst. Phys., New York

Drinkwater, M. J., Gregg, M. D., \& Hilker, M. 2003, Nature, 423, 519

Dolphin, A. E. 2000, PASP, 112, 1397

Fellhauer, M., \& Kroupa, P. 2005, MNRAS, 359, 223 
Fioc, M., \& Rocca-Volmerange, B. 1997, A\&A, 326, 950

Fioc, M., \& Rocca-Volmerange, B. 1999 [arXiv:astro-ph/9912179] (PÉGASE. 2)

Gilbert, A. M., \& Graham, J. R. 2002, in Extragalactic Star Clusters, ed. E. K. Grebel, D. Geisler, \& D. Minniti, IAU Symp., 207

González Delgado, R. M., \& Leitherer, C. 1999, ApJS, 125, 479 (GDL99)

González Delgado, R. M., Leitherer, C., Heckman, T., \& Cerviño, M. 1997, ApJ, 483, 705

González Delgado, R. M., Leitherer, C., \& Heckman, T. 1999, ApJS, 125, 489

Gray, D. F. 1981, ApJ, 251, 152

Gray, D. F. 1984, ApJ, 281, 719

Gray, D. F., \& Toner, C. G. 1987, ApJ, 322, 360

Hayes, M., Östlin, G., Mas-Hesse, J. M., et al. 2005, A\&A 438, 71

Ho, L. C., \& Filippenko, A. V. 1996a, ApJ, 466, L83

Ho, L. C., \& Filippenko, A. V. 1996b, ApJ, 472, 600

Kharchenko, N. V., Piskunov, A. E., \& Scholz, R.-D. 2004, Astron. Nachr., 325, 439

Krist, J., \& Hook, R. 2003, The Tiny Time User's Guide, version 6.1, http://www.stsci.edu/software/tinytim

Larsen, S. S., Brodie, J. P., Elmegreen B. G., et al. 2001, ApJ, 556, 801

Larsen, S. S., Brodie, J. P., \& Hunter, D. A. 2004, ApJ, 128, 2295

Leitherer, C., Schaerer, D., Goldader, J. D., et al. 1999, ApJS, 123, 3

Maoz, D., Ho, L. C., \& Sternberg, A. 2001, ApJ, 554, L139

Maraston, C., Bastian, N., Saglia, R. P., et al. 2004, A\&A, 416, 467

Massey, P., \& Hunter, D. A. 1998, ApJ, 493, 180

McCrady, N., Gilbert, A. M., \& Graham, J. R. 2003, ApJ, 596, 240

McLaughlin, D. E. 2000, ApJ, 539, 618

Mengel, S., Lehnert, M. D., Thatte, N., \& Genzel, R. 2002, A\&A, 383, 137

Meurer, G. R., Heckman, T. M., Leitherer, C., et al. 1995, AJ, 110, 2665

Meynet, G., \& Maeder, A. 2005, A\&A, 429, 581

Nidever, D. L., Marcy, G. W., Butler, R. P., Fischer, D. A., \& Vogt, S. S. 2002, ApJS, 141, 503
Ochsenbein, F. 1980, Bull. Inf. CDS, 19, 74

Ochsenbein, F., Bauer, P., \& Marcout, J. 2000, A\&AS, 143, 221

Nota, A., Pasquali, A., Drissen, L., et al. 1996, ApJS, 102, 383

Origlia, L., Leitherer, C., Aloisi, A., Greggio, L., \& Tosi, M. 2001, AJ, 122, 815

Östlin, G., Bergvall, N., \& Rönnback, J. 1998, A\&A, 335, 85

Östlin, G., Amram, P., Bergvall, N., et al. 2001, A\&A, 374, 800

Östlin, G., Zackrisson, E., Bergvall, N., \& Rönnback, J. 2003, A\&A, 408, 887

Pandey, A. K., Ogura, K., \& Sekiguchi, K. 2000, PASJ, 52, 847

Portegies Zwart, S. F., Makino, J., McMillan, S. L. W., \& Hut, P. 1999, A\&A, 348,117

Salpeter, E. 1955, ApJ, 121, 161

Scalo, J. 1998, in The Stellar Initial Mass Function, ASP Conf. Ser., 142, 201

Schaerer, D., \& Vacca, W. D. 1998, ApJ, 497, 618

Schlegel, D. J., Finkbeiner, D. P., \& Davis, M. 1998, ApJ, 500, 525

Schwartz, C. M., \& Martin, C. L. 2004, ApJ, 610, 201

Simkin, S. M. 1974, A\&A, 31, 129

Smith, L. J., \& Gallagher, J. S., III. 2001, MNRAS, 326, 1027

Spitzer, L., Jr. 1987, Dynamical Evolution of Globular Clusters, Princeton Univ. Press, Princeton, NJ

Tonry, J., \& Davis, M. 1979, AJ, 84, 1511

Turner, J. L., Beck, S. C., Crosthwaite, L. P., et al. 2003, Nature, 423, 621

Vacca, W. C., \& Conti, P. S. 1992, ApJ, 401, 543

Walcher, C. J., van der Marel, R. P., McLaughlin, D., et al. 2005, ApJ 618, 237

Werk, J. K., Jangren, A., \& Salzer, J. I. 2004, ApJ, 617, 1004

Whitmore, B. C. 2003, in A Decade of Hubble Space Telescope Science, ed. M. Livio, K. Noll, \& M. Stiavelli, Space Telescope Science Institute Symposium Series, 14, 153 (Cambridge University Press)

Whitmore, B., Heyer, I., \& Casertano, S. 1999, PASP, 111, 1559

Worthey, G., Faber, S. M., Gonzalez, J. J., \& Burstein, D. 1994, ApJ, 94, 687

Zackrisson, E., Bergvall, N., Olofsson, K., \& Siebert, A. 2001, A\&A, 375, 814 (Z01) 